\title{
Photothermal pump-probe lock-in shadowgraph technique using a thermographic camera for thermal diffusivity measurement in thin metallic filaments
}

\author{
by A. Cifuentes*, E. Marín*, S. Alvarado*, H. Cabrera**, A. Calderón ${ }^{*}$ \\ * Instituto Politécnico Nacional, Centro de Investigación en Ciencia Aplicada y Tecnología Avanzada, Legaria \\ 694, Colonia Irrigación, CP 11500, Mexico City, Mexico. Corresponding author: emarin@ipn.mx \\ ** The Abdus Salam International Centre for Theoretical Physics (ICTP), Strada Costiera 11, Trieste, Italy
}

\begin{abstract}
We present a method for measurement of the thermal diffusivity of metallic samples in the form of thin filaments, which is inspired in both the photothermal beam deflection and the shadowgraph techniques. The method utilizes the shadow projected by the sample when put against a collimated light source (a probe beam). The sample is then heated periodically by another light beam (the pump beam), giving rise to thermal waves, which propagate across it and through its surroundings. Changes in the refraction index of the surrounding media due to the heating distort the shadow. The periodical time evolution of the shadow is recorded by a FLIR SC2500 Infrared Camera and lock-in amplified in order to determine the sample's thermal diffusivity by the well-known phase slope method.
\end{abstract}

\section{Introduction}

Thermal diffusivity, $\alpha$, is one of the main parameters involved in unsteady-state heat conduction phenomena. Among the existing methods for direct determination of $\alpha$, the photothermal techniques have received considerable attention [1]. The most of these techniques are based in the common principle of periodically heating the sample with intensity modulated light and measuring the amplitude and phase-lag of the generated thermal waves. The "slope method" is based on the linear relationship that often exists between the phase-lag, $\Delta \varphi$, of the thermal wave, and the distance, $d$, to a punctual heat source at which $\Delta \varphi$ is measured. The slope of the $\Delta \varphi$ vs $d$ graph is given by $m=(\pi f / \alpha)^{1 / 2}$. From this equation the thermal diffusivity can be straightforwardly determined if the modulation frequency, $f$, is well-known.

One of the most used methods to measure $\Delta \varphi$ vs $d$ is the photothermal beam deflection (PBD) in its orthogonal skimming configuration [2]. An intensity modulated excitation or pump beam is focused onto a small spot on the sample's surface generating thermal waves that propagate to the surrounding media causing refractive index oscillations, which will cause a periodical deflection on a continuous narrow probe beam propagating parallel and very near to the sample's surface. This deflection is detected with an appropriate sensor, and $\Delta \varphi$ is recovered by lock-in phase sensitive methods.

The PBD technique has some limitations. To determine thermal diffusivity using the slope method, the offset between the probe beam and the pump beam must be adjusted and one measurement of the phase-lag must be taken for each of the desired number of points. This procedure can be quite lengthy. The perfect alignment of the probe laser beam along the sample's surface at each measurement point can be also a very difficult experimental step.

In this work we present a method that overcomes these limitations. It utilizes the shadow projected by the sample when put against a collimated light source (a probe beam). The sample is then heated periodically by another light beam (the pump beam), giving rise to thermal waves, which propagate across it and through its surroundings. Changes in the refraction index of the surrounding media due to the heating distort the shadow. The periodical variations of the shadow are recorded by a FLIR SC2500 Infrared Camera, and using its on-board incorporated lock-in capabilities the $\Delta \varphi$ vs $d$ graph is measured. The usefulness of the method is demonstrated here by simulations and measurements done in metallic test samples in the form of thin filaments.

\section{Theoretical and experimental details}

Because phase synchronous detection is an important part of the data processing, we resorted to the use of the FLIR SC2500 Infrared Camera, which has on-board incorporated lock-in capabilities. This camera has a $320 \times 256$ pixel InGaAs sensor working in the near infrared portion of the electromagnetic spectrum between 0.9 and $1.7 \mu \mathrm{m}$. Therefore, a light source emitting between this wave-length range was used as a probe beam, namely a $905 \mathrm{~nm}$ diode laser, which is collimated and widened before it is projected on the sensing element. A $445 \mathrm{~nm}$ diode laser with a $250 \mathrm{~mW}$ nominal power and a spot size of about $200 \mu \mathrm{m}$ is focused on the sample and orthogonally positioned with respect to the probe beam. The maximum frame rate of $340 \mathrm{~Hz}$ at full frame of the camera impose an upper limit of about $10 \mathrm{~Hz}$ to the maximum modulation frequency that can be used. On the other hand, the samples are immersed in a fluid with a high value of the thermophysical parameter, in our case acetonitrile, within a $1 \mathrm{~cm}^{3}$ cell placed in the trajectory of the expanded probe beam to generate the shadow. Due to the easy geometry and increasing technical interest, samples in the form of thin filaments have been analyzed in this work, although samples with other geometries can be analyzed as well [3]. The analysed materials and filaments diameters are listed in table 1. 
The shadowgraph technique is an optical technique and the mathematical equations governing it are well-known [4]. It can be shown that the change in intensity of the probe beam at the plane in which the shadow is recorded is proportional to the logarithm of the changes in the refractive index of the medium surrounding the sample, which can be calculated using its dependence on temperature and the expression for the oscillatory temperature distribution generated by the heated optically opaque filament in the surrounding fluid [5]. In this way a shadowgraph can be constructed. The phase image of this shadowgraph is shown in figure 1 (a) for a $76 \mu \mathrm{m}$ diameter platinum filament at $8 \mathrm{~Hz}$ modulation frequency. When analyzed at distances close to the object's surface, it has been concluded that the linear relationship between $\Delta \varphi$ and $d$ is guaranteed only if a thermal diffusivity of the sample is higher than that of the surrounding medium. Therefore, the applicability of the method is limited to good heat conductors.

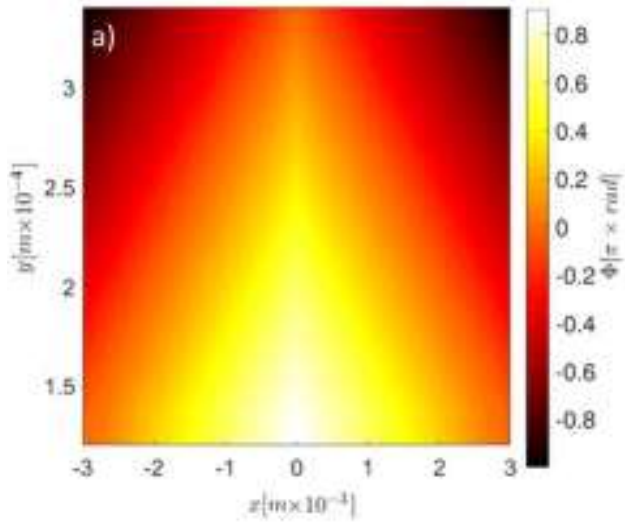

a)

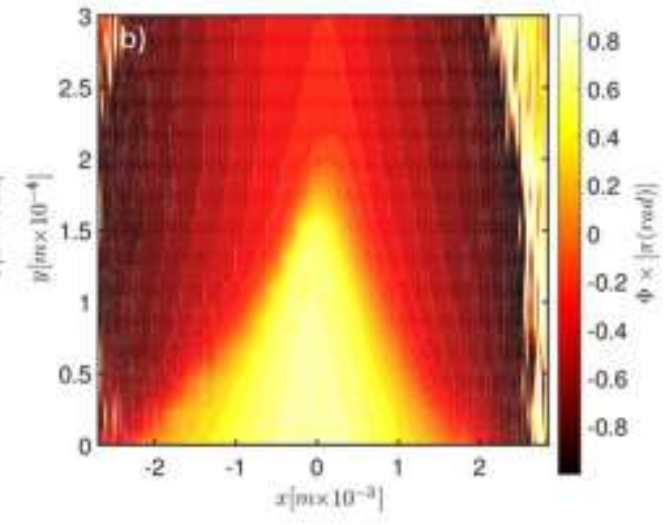

b)

Fig. 1: a) Phase imaging of the simulated shadowgraph for a $76 \mu \mathrm{m}$ diameter Pt filament. b) Experimental obtained shadowgraph for the same sample. The modulation frequency was $8 \mathrm{~Hz}$.

\section{Experimental results and conclusions}

Figure 1 (b) shows a typical experimental result (phase image) for a Pt filament with diameter $76 \mu \mathrm{m}$ at $8 \mathrm{~Hz}$. It can be seen the similitude with the shadowgraph obtained with the computer simulation. The $\Delta \varphi$ vs $d$ curve is then obtained for the pixels closest to the filament surface. From the best least squares linear fit thermal diffusivity is calculated using the slope method described in section 1. Table 1 shows the results obtained for this and other samples. A good agreement is observed with literature reported values.

Table 1. Experimental results. The modulation frequency was $8 \mathrm{~Hz}$.

\begin{tabular}{|l|l|}
\hline Material / diameter $(\mu \mathrm{m})$ & $\alpha\left(\mathrm{m}^{2} / \mathrm{s}\right) \times 10^{-5}$ \\
\hline $\mathrm{Cu} / 125$ & $12.0 \pm 0.4$ \\
\hline $\mathrm{Pt} / 76$ & $2.4 \pm 0.1$ \\
\hline $\mathrm{Zn} / 125$ & $4.2 \pm 0.2$ \\
\hline $\mathrm{PbSn}(40 / 60) / 1000$ & $3.3 \pm 0.1$ \\
\hline
\end{tabular}

In conclusion, the shadowgraph technique was successfully used to obtain the thermal diffusivity of different filamentary samples through the phase slope method, making it a promising alternative to currently established methods. The technique could also be used to study other sample geometries and sample fluid interactions.

\section{REFERENCES}

[1] Almond S D P and Patel PM. Photothermal Science and Techniques Netherlands: Springer; 1996.

[2] Salazar A, Sanchez-Lavega A and Fernandez J. Thermal diffusivity measurements in solids by the "mirage" technique: Experimental results. J. Appl. Phys. 1991; 69: 1216-1223.

[3] Cifuentes M A, Alvarado S, Cabrera H, Calderón A, Marín E. Thermal Diffusivity Measurement by Lock-in Photothermal Shadowgraph Method. J. of Appl. Phys. 2016; 119: 164902-164907

[4] Settles G. S. Schlieren and Shadowgraph Techniques Berlin Heidelberg: Springer; 2001.

[5] Salazar A, Mendioroz A, Fuente-Dacal R and Celorrio R. Accurate measurements of the thermal diffusivity of thin filaments by lock-in thermography. J. Appl. Phys. 2010; 4: 043508-043514. 